\title{
The prognosis for patients with newly diagnosed glioblastoma receiving bevacizumab combination therapy: a meta-analysis
}

This article was published in the following Dove Press journal:

OncoTargets and Therapy

\section{Ke-Li Liao' \\ Song Huang' \\ Yu-Peng $\mathrm{Wu}^{2}$}

'Department of Neurosurgery, Zigong First People's Hospital, Zigong, Sichuan, People's Republic of China; ${ }^{2}$ Department of Neurosurgery,

The Second Hospital of Hebei Medical University, Shijiazhuang, Hebei, People's Republic of China
Correspondence: Yu-Peng Wu Department of Neurosurgery, The Second Hospital of Hebei Medical University, 215 West Heping Road, Shijiazhuang, Hebei 050000, People's Republic of China Tel +86 I5530I0 760I Email irdI3v@163.com
Background: A combination of temozolomide (TMZ) and radiotherapy and subsequent adjuvant chemotherapy is the gold standard of treatment for glioblastoma (GB). Bevacizumab (BEV), a humanized monoclonal antibody that blocks the effects of vascular endothelial growth factor A, has produced impressive response rates for recurrent GB and has been approved as second-line therapy. The efficacy and safety of BEV in newly diagnosed GB are not known.

Aim: This systematic meta-analysis was undertaken to evaluate the value of combination therapy involving BEV in newly diagnosed GB.

Methods: Electronic databases were searched for eligible literature up to October 2017. Randomized controlled trials assessing the efficacy and safety of BEV in patients with newly diagnosed GB were included, of which the main outcomes were progression-free survival (PFS), overall survival (OS), and adverse events (AEs). All the data were pooled with the corresponding 95\% confidence intervals (CIs) using RevMan software. Sensitivity analyses and heterogeneity were quantitatively evaluated.

Results: A total of six randomized controlled trials were included in this analysis. The experimental BEV group had significantly improved the overall PFS $(\mathrm{OR}=0.46,95 \% \mathrm{CI}=0.26-0.81$, $P=0.007)$, as well as PFS at 6 months $(\mathrm{OR}=3.47,95 \% \mathrm{CI}=2.85-4.22, P<0.00001)$ and $\mathrm{PFS}$ at 12 months ( $\mathrm{OR}=2.02,95 \% \mathrm{CI}=1.66-2.46, P<0.00001)$, respectively. However, there were no significant differences in PFS at 24 months with $\mathrm{BEV}(\mathrm{OR}=0.95,95 \% \mathrm{CI}=0.61-1.48, P=0.82)$. OS at 6 months $(P=0.07)$ and 24 months $(P=0.07)$ was not significantly improved with BEV in patients with newly diagnosed GB. However, the meta-analysis on the OS at 12 months showed differences with $\mathrm{BEV}(\mathrm{OR}=1.24,95 \% \mathrm{CI}=1.03-1.50, P=0.02)$.

Conclusion: Our study indicates that addition of BEV for newly diagnosed GB resulted in a superior PFS rate. However, the combination therapy involving BEV did not improve OS. Future investigations are needed to analyze whether BEV helps improve OS efficacy.

Keywords: bevacizumab, glioblastoma, newly diagnosed, meta-analysis, neoadjuvant

\section{Introduction}

Glioblastoma (GB) is the most aggressive brain malignancy in adults. ${ }^{1}$ Even with the advances in imaging and available standard treatment, the survival and quality of life (QoL) of patients with GB still remain poor. ${ }^{2,3}$ When GB is diagnosed, the median overall survival (OS) with a combination of conventional radiation and temozolomide (TMZ) is only 14 months after diagnosis. ${ }^{3}$ Therefore, it is necessary to search for more effective treatment options for newly diagnosed GB.

GB is a highly vascularized tumor with upregulated vascular endothelial growth factor (VEGF), which inhibits tumor growth and progression. ${ }^{4,5}$ As an antiangiogenic 
(anti-VEGF) agent, bevacizumab (BEV) serves as a potentially therapeutic option for GB. ${ }^{6}$ Based on the promising outcomes of two Phase II trials, the US Food and Drug Administration (FDA) has approved BEV in the second-line treatment of recurrent GB in 2009. ${ }^{14}$ The addition of BEV produces impressive progression-free survival (PFS) and response rate compared to standard treatment for recurrent $\mathrm{GB}$, while the value of BEV on OS is still a matter of debate..$^{7-9}$

At the time of the approval of BEV in recurrent disease, several randomized controlled trials (RCTs) were launched to investigate the value of $\mathrm{BEV}$ in addition to the treatment for newly diagnosed GB. ${ }^{10-15}$ Treatment combined with BEV exhibited significant activity in PFS for newly diagnosed GB patients, while further evaluation is needed to assess disease progression after antiangiogenic therapy.

In order to make care for newly diagnosed GB more rational, we conducted a meta-analysis of RCTs to evaluate the therapeutic value of BEV compared with standard therapy (ST).

\section{Methods}

\section{Search strategy}

Two investigators independently searched the electronic databases PubMed, Embase, and Cochrane library up to October 2017. We searched for all randomized clinical trials evaluating the value of BEV in patients with newly diagnosed GB. The process involved finding all articles with the keywords "bevacizumab" AND "glioblastoma" AND "newly diagnosed" AND "efficacy", and the relevant Medical Subject Heading (MeSH) terms were searched. The reference lists of all articles that dealt with the topic of interest were also hand-searched to check for additional relevant publications. The search was restricted to trials published in the English language.

\section{Eligibility criteria}

Studies that met the following criteria were included in the meta-analysis: 1) the studies were designed as RCTs; 2) studies focused on newly diagnosed GB treated with BEV; 3) the outcomes of interest regarding the treatment efficacy (survival), safety (adverse events [AEs]), and hazard ratios (HRs) with corresponding $95 \%$ CIs were provided; 4) only the full texts were included. The studies that did not meet the above inclusion criteria were excluded from the meta-analysis.

\section{Quality assessment}

Study quality was assessed using the Jadad seven-item scale recommended by The Cochrane Handbook for Systematic Reviews of Interventions. The Jadad seven-item scale was introduced to evaluate the overall methodological quality of RCTs.

\section{Data extraction}

The data extraction was conducted independently by two authors. Disagreement was revolved by consensus. The main characteristics extracted from the selected studies were the following: first author family name, publication year, trial name, study design, sample size, and the outcomes of interest (AEs, PFS, and OS). The corresponding hazard ratios (HRs) with 95\% confidence intervals (CIs) were used to describe the main outcomes of the studies, including OS, PFS, and AE data, and 95\% CIs were calculated for each estimate.

\section{Statistical analysis}

The main outcomes of the studies were OS, PFS, and AEs. If HRs and corresponding 95\% CIs were reported, the lnHRs and the corresponding $\ln$ lower limits and $\ln$ upper limits were used as data points in pooling analysis. While, if the study did not provide HRs or $95 \%$ CIs, the only available data were in the form of Kaplan-Meier (K-M) curves. Survival data were extracted from the form of the $\mathrm{K}-\mathrm{M}$ survival curve, according to the methods described by Tierney et al. ${ }^{16}$ The $I^{2}$ statistical test was used to further examine statistical heterogeneity between the trials. ${ }^{17}$ Studies with an $I^{2} \geq 50 \%$ were considered to exhibit moderate and high heterogeneity, and those with $I^{2}<50 \%$ were considered to have low heterogeneity. ${ }^{18}$ Summary HRs were calculated by using fixed-effect models when there was low heterogeneity among studies. Otherwise, random-effect models were used. A $P$-value $<0.05$ was considered to be statistically significant. All analyses were conducted with Review Manager Version 5.3 software (Revman; The Cochrane Collaboration, Oxford, UK). Findings of our meta-analysis are shown in forest plots. The Begg's test and the Egger's test were used to evaluate publication bias.

\section{Results}

\section{Search results and characteristics of studies}

A total of 214 studies were retrieved initially for evaluation. Based on the criteria described in the "Methods" section, 10 publications were evaluated in more detail, but some did not provide enough detail of the outcomes of two approaches. Therefore, we had a final total of six RCTs ${ }^{10-15}$ assessing the value of BEV in patients with newly diagnosed GB. The search process is described in Figure 1.

All included papers in this study were based on moderateto high-quality evidence. Table 1 describes the primary characteristics of the eligible studies in more detail. 


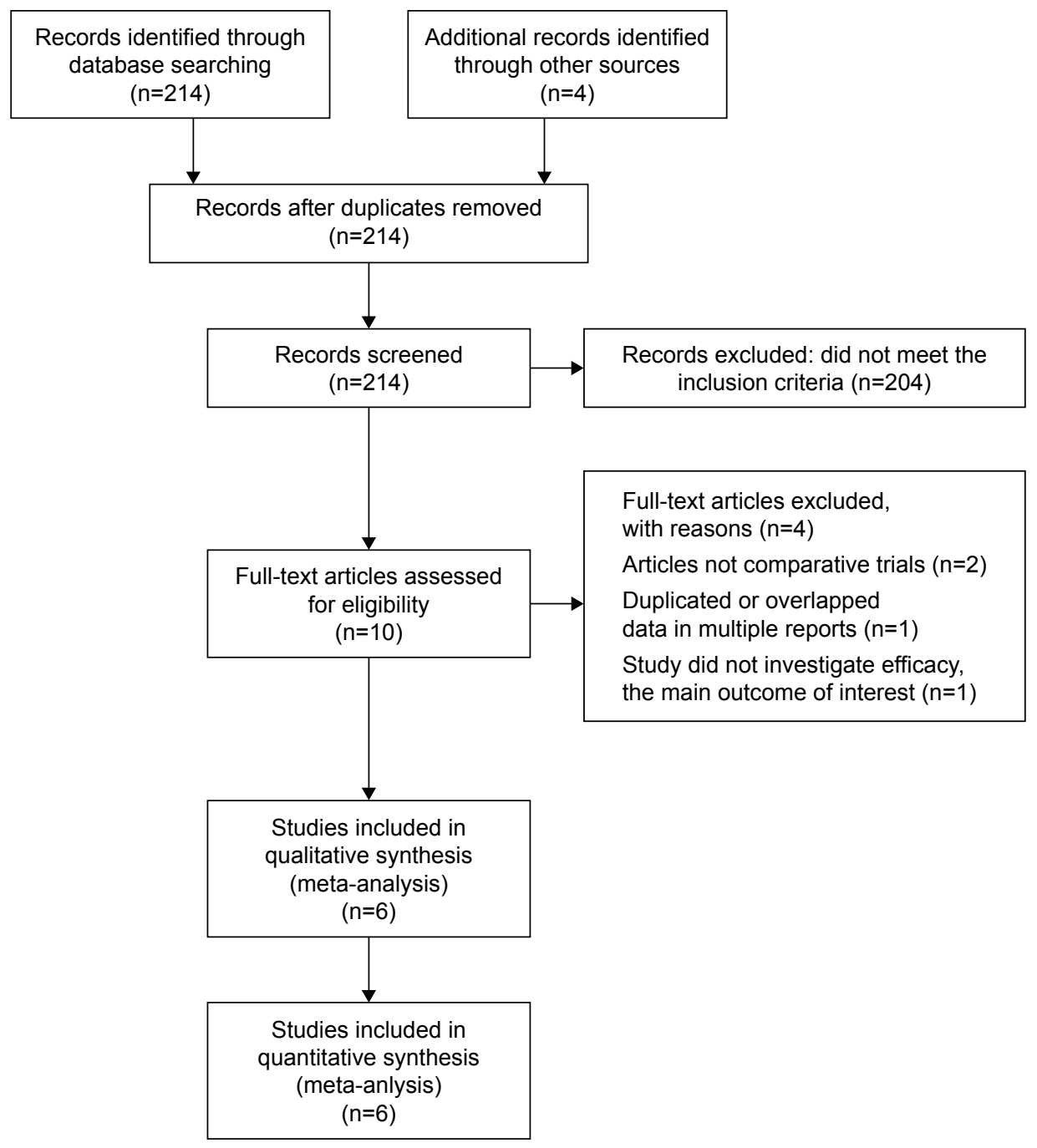

Figure I PRISMA flow chart of selection process to identify studies eligible for pooling. Abbreviation: PRISMA, Preferred Reporting Items for Systematic Reviews and Meta-Analyses.

\section{Clinical and methodological heterogeneity}

Pooled analysis of PFS comparing the addition of BEV with the control group

Pooling the PFS data from five studies ${ }^{10,11,13-15}$ showed that BEV prolonged the PFS (HR $=0.69,95 \% \mathrm{CI}=0.63-0.77$, $P<0.00001$ ) compared with the control group (Figure 2).
Subgroup analysis of PFS comparing the addition of BEV with the control group

Overall, the six studies that reported data on PFS at different months are shown in Figures 3-5. Pooled data showed that the PFS data achieved advantage with BEV agents, with the pooled OR being 3.47 (95\% CI 2.85-4.22, $P<0.00001)$ at 6 months (Figure 3$) ; 2.02(95 \%$ CI 1.66-2.46, $P<0.00001)$ at

Table I The primary characteristics of the eligible studies in more detail

\begin{tabular}{|c|c|c|c|c|c|c|c|c|}
\hline \multirow[t]{2}{*}{ Study } & \multirow[t]{2}{*}{ Trials } & \multicolumn{2}{|c|}{ Mean age, years } & \multicolumn{2}{|l|}{ Cases, $\mathbf{n}$} & \multicolumn{2}{|l|}{ Treatment } & \multirow{2}{*}{$\begin{array}{l}\text { Follow-up } \\
\text { (months) }\end{array}$} \\
\hline & & Experiment & Control & Experiment & Control & Experiment & Control & \\
\hline Gilbert et al, $2014^{13}$ & AVAglio & NA & NA & 312 & 309 & $\mathrm{BEV}+\mathrm{RT}+\mathrm{TMZ}$ & $\mathrm{RT}+\mathrm{TMZ}$ & 30 \\
\hline Chinot et al, $2014^{14}$ & RTOG 082512 & 57 & 56 & 458 & 463 & $\mathrm{BEV}+\mathrm{RT}+\mathrm{TMZ}$ & $\mathrm{RT}+\mathrm{TMZ}$ & 32 \\
\hline Chauffert et al, 2014"1 & TEMAVIR & 60.2 & 60.9 & 60 & 60 & $\mathrm{BEV}+\mathrm{RT}+\mathrm{TMZ}$ & $\mathrm{RT}+\mathrm{TMZ}$ & 24 \\
\hline Herrlinger et al, $2016^{15}$ & GLARIUS & 56 & 56 & 116 & 54 & $\mathrm{BEV}+\mathrm{RT}+\mathrm{TMZ}+\mathrm{IRI}$ & $\mathrm{RT}+\mathrm{TMZ}$ & 36 \\
\hline Carlson et al, $2015^{12}$ & NA & 55.9 & 59.5 & 30 & 26 & IMRT+BEV+TMZ & IMRT+TMZ & 60 \\
\hline Balana et al, $2016^{10}$ & GENOM 009 & 62.9 & 62 & 48 & 45 & $B E V+R T+T M Z$ & $\mathrm{RT}+\mathrm{TMZ}$ & NA \\
\hline
\end{tabular}

Abbreviations: BEV, bevacizumab; IMRT, intensity-modulated radiotherapy; IRI, irinotecan; NA, not available; RT, radiotherapy; TMZ, temozolomide. 


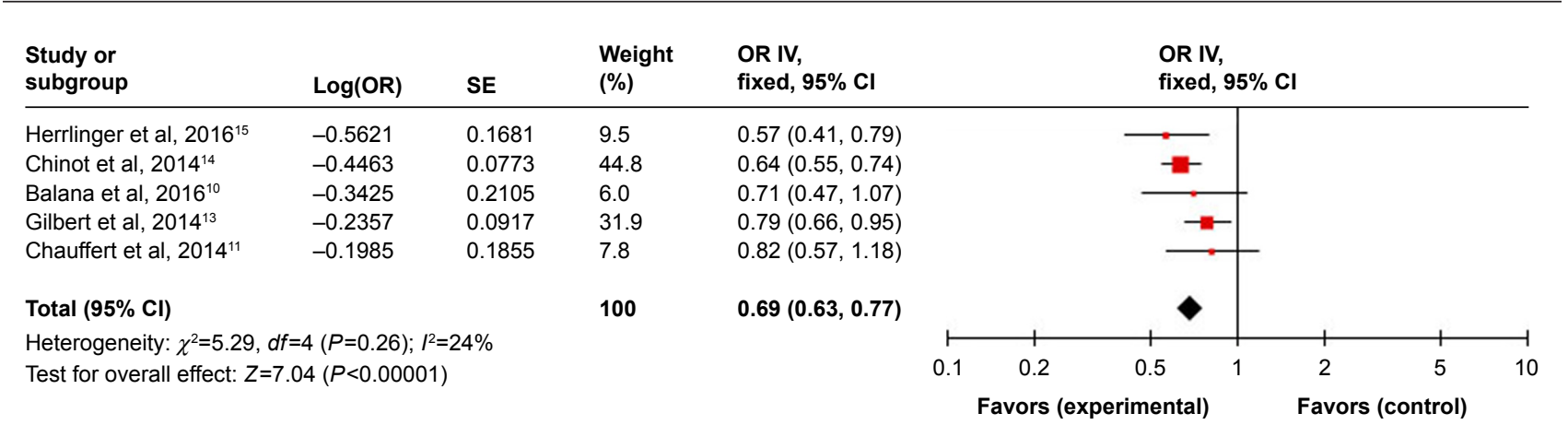

Figure 2 Pooled analysis of PFS comparing the bevacizumab-addition group with the control group.

Abbreviations: $\mathrm{Cl}$, confidence interval; IV, inverse-variance; OR, odds ratio; PFS, progression-free survival; SE, standard error.

12 months (Figure 4); and 0.95 (95\% CI 0.61-1.48, $P=0.82$ ) at 24 months (Figure 5). In other words, the addition of BEV agents increases the PFS.

\section{Pooled analysis of OS comparing the addition of BEV} with the control group

A random-effects model was used to pool the OS data. ${ }^{10,11,13-15}$ The pooled data showed that a BEV agent plus chemotherapy did not improve the OS (HR $=0.91,95 \% \mathrm{CI}=0.76-1.08$, $P=0.28$ ) than the control treatment (Figure 6).

\section{Subgroup analysis of OS comparing the addition of $\mathrm{BEV}$ with the control group}

Subgroup analysis of OS data at different months was available for all six RCTs. ${ }^{10-15}$ Analysis showed that the results of OS at 6 months (HR $=1.28,95 \% \mathrm{CI}=0.98-1.67, P=0.07$ ) (Figure 7) with BEV were no longer significant in patients with newly diagnosed GB, while the meta-analysis on the $\mathrm{OS}$ at 12 months showed differences with BEV ( $\mathrm{HR}=1.24$, $95 \%$ CI $=1.03-1.50, P=0.02$ ) (Figure 8). However, OS at 24 months still did not reach statistically significant difference ( $\mathrm{HR}=1.22,95 \% \mathrm{CI}=0.98-1.52, P=0.07$ ) (Figure 9).

\section{Pooled analysis of AEs comparing the addition of} $\mathrm{BEV}$ with the control group

Due to the limited data in all studies, systematic evaluations of $\mathrm{AE}$ data were not possible in this meta-analysis.
Gilbert et $\mathrm{al}^{13}$ report that toxicities, hypertension, thromboembolic events, intestinal perforation, and neutropenia were observed in the BEV group. Over time, patients treated with BEV have an increased symptom burden following a worse QoL, as well as debilitating neurological symptoms. In Chinot et al, ${ }^{14}$ grade 3 or higher AEs (66.8\% vs $\left.51.3 \%\right)$ were more frequent in the BEV group.

\section{Discussion}

GB is the most frequent malignant brain tumor with a poor prognosis. Patients with recurrent GBs have a poor OS, and available therapies have a limited impact on prognosis. Therefore, development of a new approach is essential to improve the outcomes in patients with newly diagnosed GB. In Phase III RCTs, ${ }^{3}$ the addition of TMZ to chemoradiotherapy (CRT) and subsequent adjuvant chemotherapy has been the standard therapy with newly diagnosed GB and is thought to be the backbone for further understanding therapy choices. ${ }^{19}$ Research reports have shown that GB has multistep cytostatic effects that alter neovascularization of brain tissue to form new blood vessels, which may help slow tumor progression and proliferation..$^{20,21}$ VEGF is overexpressed in malignant gliomas and has been used as a therapeutic target for brain tumors. ${ }^{22}$

BEV, a humanized monoclonal antibody against the VEGF ligand, has received FDA approval for recurrent

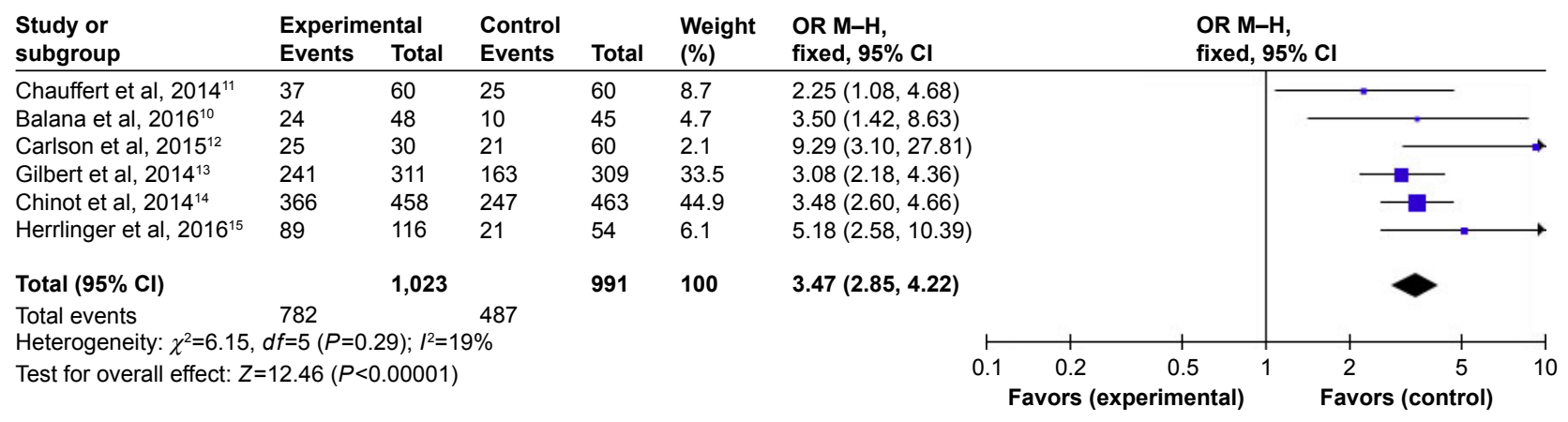

Figure 3 Pooled analysis of PFS at 6 months comparing the bevacizumab-addition group with the control group. Abbreviations: $\mathrm{Cl}$, confidence interval; $\mathrm{M}-\mathrm{H}$, Mantel-Haenszel; OR, odds ratio; PFS, progression-free survival. 


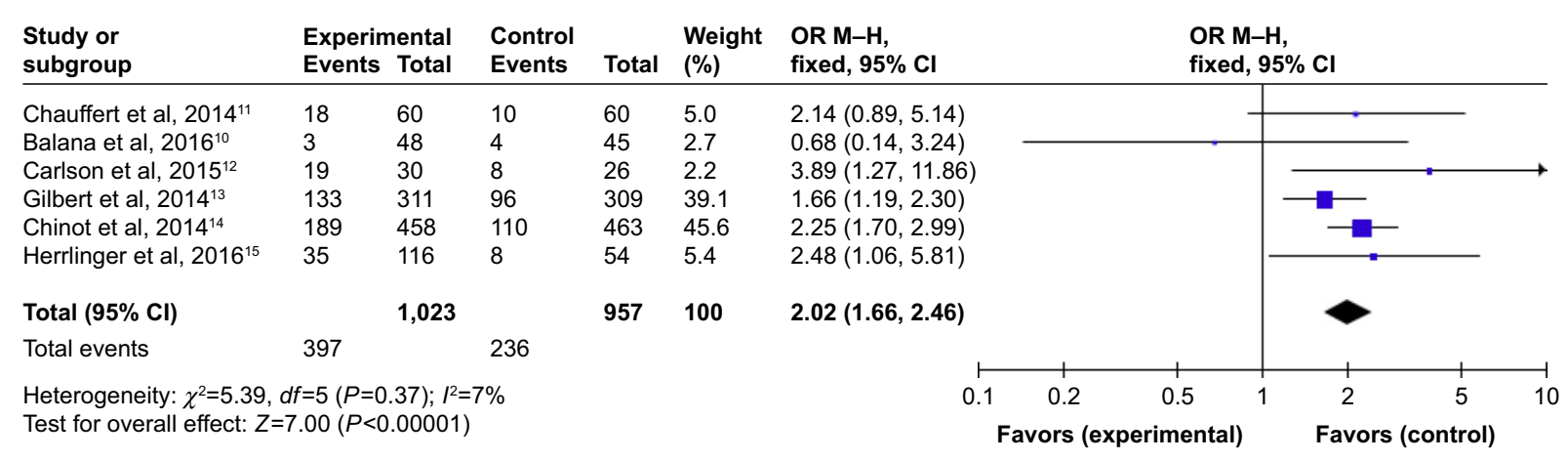

Figure 4 Pooled analysis of PFS at 12 months comparing the bevacizumab-addition group with the control group. Abbreviations: $\mathrm{Cl}$, confidence interval; M-H, Mantel-Haenszel; OR, odds ratio; PFS, progression-free survival.

GBMs in 2009..$^{23-25}$ The results have suggested that a potential benefit could be achieved by BEV for newly diagnosed GB. ${ }^{26}$ To assess whether BEV would be safe and effective for the treatment of newly diagnosed GB, we conducted a meta-analysis to evaluate the value of therapy with the combination of BEV.

All included studies chose OS and PFS as the primary end points. At the time of disease progression, crossover regimen may continue to benefit patients following an initial response to therapy with or without BEV. Therefore, in newly diagnosed GBs, the end point of PFS has beneficial effects in evaluating the potential effects of combined treatment with BEV than OS. ${ }^{13}$

Data from the trial of Carlson et al, ${ }^{12}$ showed that compared with placebo plus TMZ and radiotherapy, the combination using BEV did not improve OS, but increases PFS, although it is statistically nonsignificant $(P=0.39)$. In the AVAglio and RTOG 0825 studies, ${ }^{13,14}$ the PFS was significantly improved with the addition of BEV, but OS did not show benefit.

In this study, we conclude that the combination of BEV for newly diagnosed GB is beneficial in terms of prolonging median PFS but not OS. Our results did not indicate any benefit from BEV for newly diagnosed GB in terms of median
OS. The AVAglio, RTOG 082512, and GLARIUS ${ }^{13-15}$ trials obtained similar results. In our analysis, the pooled analysis did not show that the PFS benefit translates into OS prolongation. As potential reasons for this observation, patients with GB exhibited worse neurocognition and poor survival rate with prolonged use of BEV, which might be caused by BEV resistance. Resistance to chemotherapy was considered to influence the effectiveness of BEV treatment for GB. As an antiangiogenic (anti-VEGF) agent, BEV has been investigated as complementary to standard chemotherapy to suppress tumor growth. ${ }^{27}$ Due to the different angiogenesis pathways of the $V E G F$ genes, there may be benefit in continuing BEV treatment even after resistance to chemotherapy. ${ }^{28-30}$ However, the potential antitumor effects underlying resistance to antiangiogenic agents are yet to be fully evaluated. The point of molecular signatures may reveal subsets of GBs that are particularly sensitive or resistant to BEV. In additional analyses of subgroups of patients based on different genetic mutations, we may identify patients who had a selected survival benefit response to BEV. Furthermore, BEV's radiographic effect has been reported to be associated with an increased incidence of PFS. BEV stabilizes the blood-brain barrier, minimizing the ability of the magnetic resonance imaging (MRI) contrast agent gadolinium to reach the tumor, thus

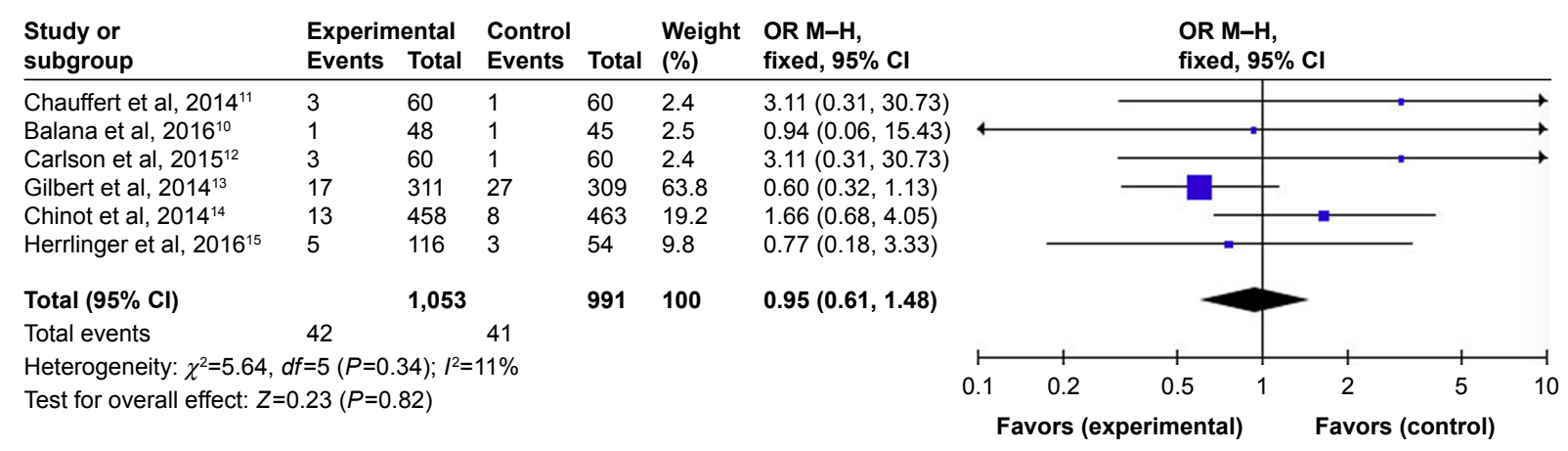

Figure 5 Pooled analysis of PFS at 24 months comparing the bevacizumab-addition group with the control group.

Abbreviations: $\mathrm{Cl}$, confidence interval; $\mathrm{M}-\mathrm{H}$, Mantel-Haenszel; OR, odds ratio; PFS, progression-free survival. 


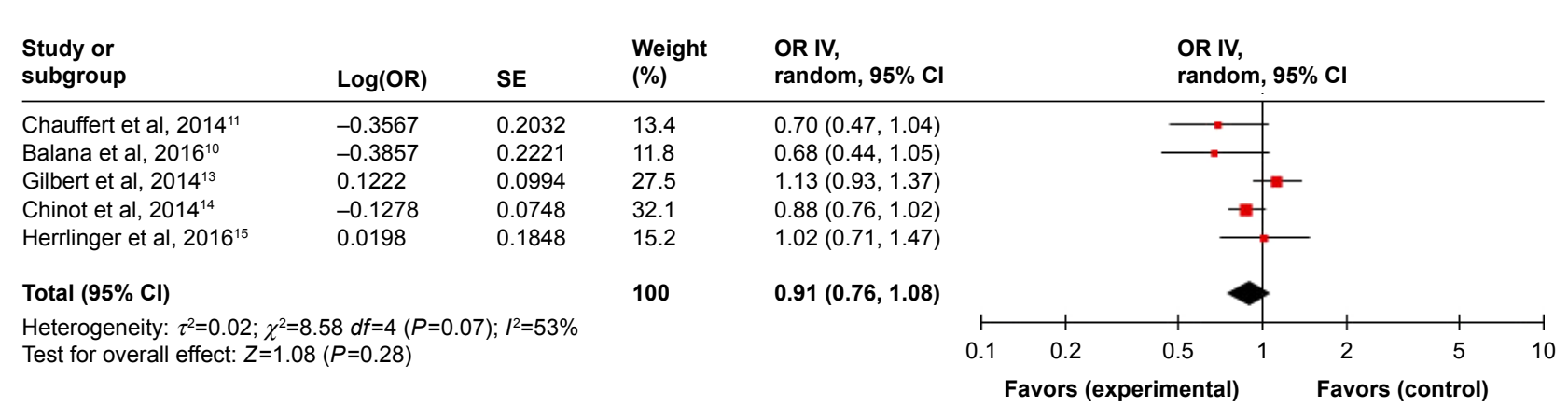

Figure 6 Pooled analysis of OS comparing the bevacizumab-addition group with the control group.

Abbreviations: $\mathrm{Cl}$, confidence interval; IV, inverse-variance; OR, odds ratio; OS, overall survival; SE, standard error.

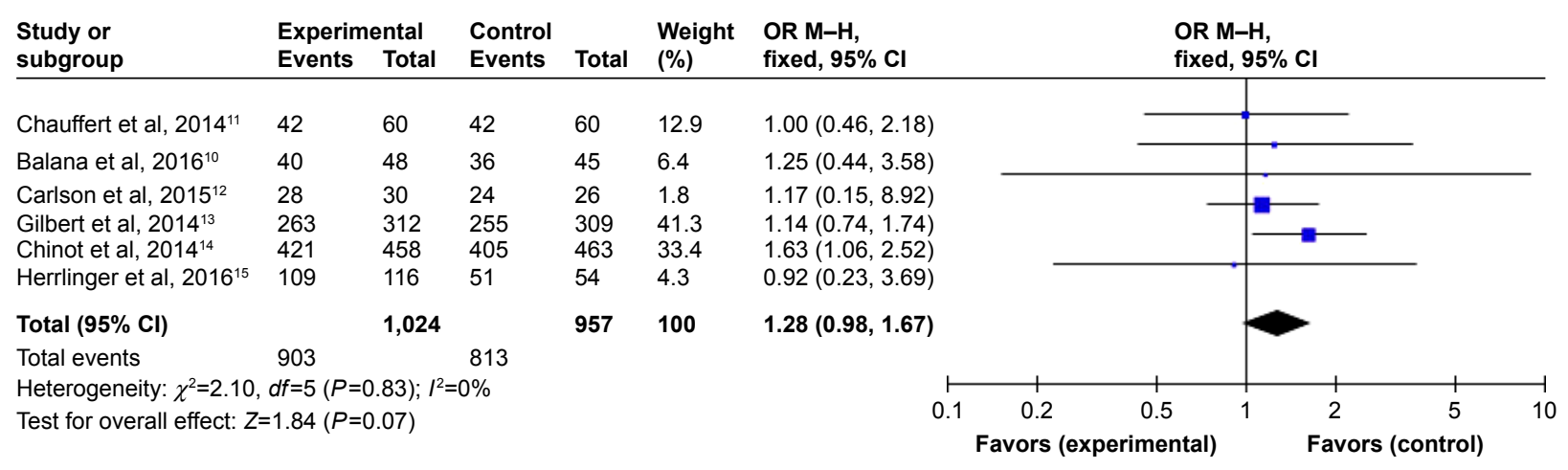

Figure 7 Pooled analysis of OS at 6 months comparing the bevacizumab-addition group with the control group.

Abbreviations: $\mathrm{Cl}$, confidence interval; $\mathrm{M}-\mathrm{H}$, Mantel-Haenszel; OR, odds ratio; OS, overall survival.

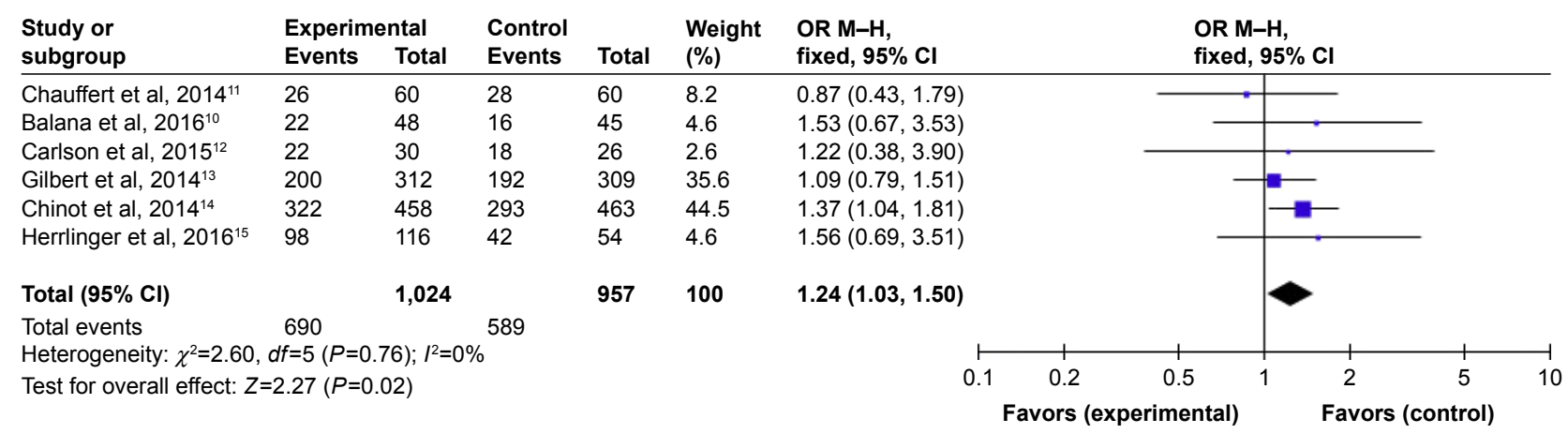

Figure 8 Pooled analysis of OS at 12 months comparing the bevacizumab-addition group with the control group.

Abbreviations: $\mathrm{Cl}$, confidence interval; $\mathrm{M}-\mathrm{H}$, Mantel-Haenszel; OR, odds ratio; OS, overall survival.

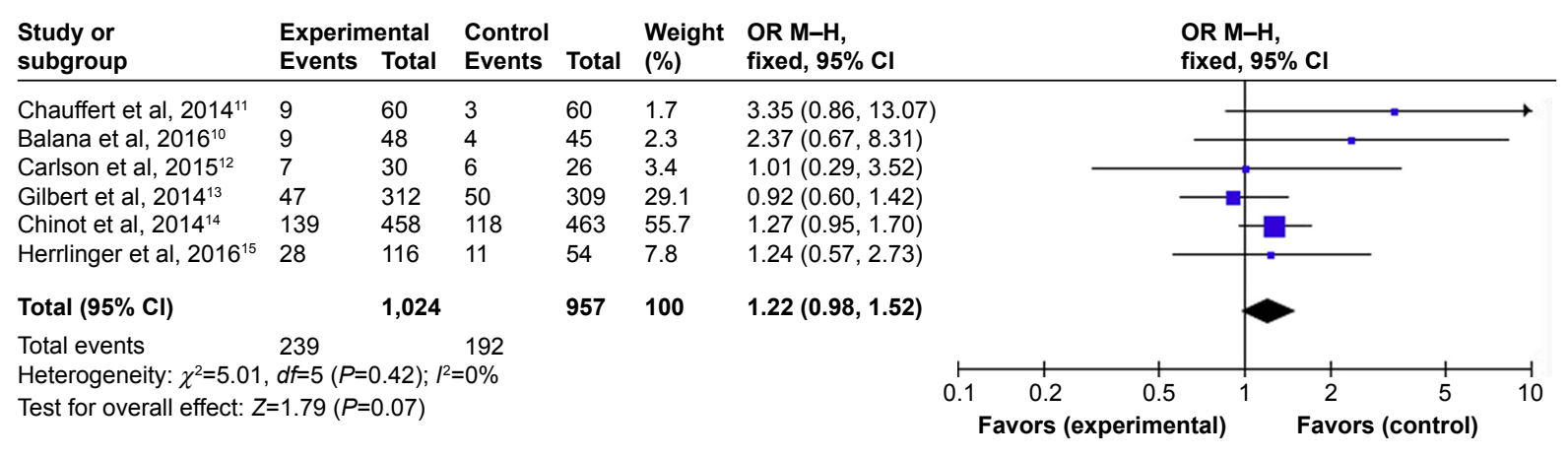

Figure 9 Pooled analysis of OS at 24 months comparing the bevacizumab-addition group with the control group.

Abbreviations: $\mathrm{Cl}$, confidence interval; $\mathrm{M}-\mathrm{H}$, Mantel-Haenszel; OR, odds ratio; OS, overall survival. 
showing "improved" or "cleaner" MRIs, hence delaying the diagnosis of progression (a largely radiographic diagnosis) and consequently showing prolonged PFS. Moreover, secondline crossover BEV therapy has been shown to play an important role in the OS. Therefore, there is much more detailed knowledge on postprogression therapy. In GLARIUS, ${ }^{15}$ patients who received the crossover BEV therapies achieved a significant OS benefit compared with other BEV first-line trials. The RPSFT analysis, which evaluated the influence of crossover BEV treatment, suggested a significant OS benefit of the combination therapy of BEV and thus indicates that $\mathrm{BEV}$ crossover may be associated with OS prolongation. ${ }^{15}$

To date, the mechanism of long-term BEV treatment has not been established. Further studies of other physiological molecularly defined subgroups may suggest a potential marker panel for BEV, which would need more clinical trials to clarify.

In previous studies, the serious AEs observed more frequently in the BEV group included abdominal pain, headache, fatigue, hypertension, diarrhea, neutropenia, complications of wound healing, cerebral hemorrhage or ischemia, gastrointestinal perforation, congestive heart failure, and anemia. ${ }^{13,14}$ Due to the limited data shown in all studies, systematic evaluations of AE data were not possible in this meta-analysis. In a previous meta-analysis, BEV therapy was not found to be associated with serious AEs for newly diagnosed GB. ${ }^{31}$ However, the authors did indicate a trend toward significance with respect to BEV treatment. ${ }^{31}$

In this systematic analysis assessing the value of BEV in the treatment of newly diagnosed GB, there are some limitations that should not be ignored. First, as only full texts were included and this study was a study-level meta-analysis, which resulted in imbalance between the two groups, clinical heterogeneity among trials should be taken into consideration in the interpretation of our findings. Second, most included studies reported short-term survival rates within 2 years of follow-up. There were insufficient data to determine long-term survival rate. Further studies are needed to report survival rates at 3 years or longer follow-ups. Third, as the data on AEs in the included trials is limited, we did not perform the analysis of AEs in this meta-analysis.

\section{Conclusion}

$\mathrm{BEV}$ treatment has a potential benefit in terms of PFS, but not OS. The apparent lack of OS benefit is probably associated with the confounding effects of crossover, although many other explanations are possible. On the basis of all available studies (AVAglio, RTOG 0825, and GLARIUS), ${ }^{13-15}$
BEV therapy does not have a place in ST with newly diagnosed GB. We suggest that studies of correlative molecular signatures are needed to identify particular subgroups of patients who will receive benefit from the combination of $\mathrm{BEV}$. Further research is needed to define the best treatment response with the lowest possible toxicity in selecting suitable patients with consideration for their complications and treatment regimen.

\section{Disclosure}

The authors report no conflicts of interest in this work.

\section{References}

1. Stupp R, Pavlidis N, Jelic S, Force EGT. ESMO minimum clinical recommendations for diagnosis, treatment and follow-up of malignant glioma. Ann Oncol. 2005;16(Suppl 1):i64-i65.

2. Norden AD, Drappatz J, Wen PY. Novel anti-angiogenic therapies for malignant gliomas. Lancet Neurol. 2008;7(12):1152-1160.

3. Stupp R, Hegi ME, Mason WP, et al. Effects of radiotherapy with concomitant and adjuvant temozolomide versus radiotherapy alone on survival in glioblastoma in a randomised phase III study: 5-year analysis of the EORTC-NCIC trial. Lancet Oncol. 2009;10(5):459-466.

4. Birner P, Piribauer M, Fischer I, et al. Vascular patterns in glioblastoma influence clinical outcome and associate with variable expression of angiogenic proteins: evidence for distinct angiogenic subtypes. Brain Pathol. 2003;13(2):133-143.

5. Godard S, Getz G, Delorenzi M, et al. Classification of human astrocytic gliomas on the basis of gene expression: a correlated group of genes with angiogenic activity emerges as a strong predictor of subtypes. Cancer Res. 2003;63(20):6613-6625.

6. Wen PY, Brandes AA. Treatment of recurrent high-grade gliomas. Curr Opin Neurol. 2009;22(6):657-664.

7. Sepulveda JM, Belda-Iniesta C, Gil-Gil M, et al. A phase II study of feasibility and toxicity of bevacizumab in combination with temozolomide in patients with recurrent glioblastoma. Clin Transl Oncol. 2015; 17(9):743-750.

8. Maree K, Simes J, Wheeler H, et al. A randomised phase II study of carboplatin and bevacizumab in recurrent glioblastoma multiforme (CABARET). Presented at: 2013 American Society of Clinical Oncology Annual Meeting; May 31-June 4; 2013; Chicago, IL, USA.

9. Taal W, Oosterkamp HM, Walenkamp AM, et al. Single-agent bevacizumab or lomustine versus a combination of bevacizumab plus lomustine in patients with recurrent glioblastoma (BELOB trial): a randomised controlled phase 2 trial. Lancet Oncol. 2014;15(9):943-953.

10. Balana C, De Las Penas R, Sepulveda JM, et al. Bevacizumab and temozolomide versus temozolomide alone as neoadjuvant treatment in unresected glioblastoma: the GENOM 009 randomized phase II trial. J Neurooncol. 2016;127(3):569-579.

11. Chauffert B, Feuvret L, Bonnetain F, et al. Randomized phase II trial of irinotecan and bevacizumab as neo-adjuvant and adjuvant to temozolomide-based chemoradiation compared with temozolomidechemoradiation for unresectable glioblastoma: final results of the TEMAVIR study from ANOCEFdagger. Ann Oncol. 2014;25(7): 1442-1447.

12. Carlson JA, Reddy K, Gaspar LE, et al. Hypofractionated-intensity modulated radiotherapy (hypo-IMRT) and temozolomide (TMZ) with or without bevacizumab (BEV) for newly diagnosed glioblastoma multiforme (GBM): a comparison of two prospective phase II trials. J Neurooncol. 2015;123(2):251-257.

13. Gilbert MR, Dignam JJ, Armstrong TS, et al. A randomized trial of bevacizumab for newly diagnosed glioblastoma. N Engl J Med. 2014; 370(8):699-708. 
14. Chinot OL, Wick W, Mason W, et al. Bevacizumab plus radiotherapytemozolomide for newly diagnosed glioblastoma. $N \mathrm{Engl} \mathrm{J} \mathrm{Med.}$ 2014;370(8):709-722.

15. Herrlinger U, Schafer N, Steinbach JP, et al. Bevacizumab plus irinotecan versus temozolomide in newly diagnosed O6-methylguanineDNA methyltransferase nonmethylated glioblastoma: the randomized GLARIUS trial. J Clin Oncol. 2016;34(14):1611-1619.

16. Tierney JF, Stewart LA, Ghersi D, Burdett S, Sydes MR. Practical methods for incorporating summary time-to-event data into meta-analysis. Trials. 2007;8:16.

17. Higgins JP, Thompson SG. Quantifying heterogeneity in a metaanalysis. Stat Med. 2002;21(11):1539-1558.

18. Higgins JP, Thompson SG, Deeks JJ, Altman DG. Measuring inconsistency in meta-analyses. BMJ. 2003;327(7414):557-560.

19. Stupp R, Mason WP, van den Bent MJ, et al. Radiotherapy plus concomitant and adjuvant temozolomide for glioblastoma. $N$ Engl J Med. 2005;352(10):987-996.

20. Stupp R, van den Bent MJ, Hegi ME. Optimal role of temozolomide in the treatment of malignant gliomas. Curr Neurol Neurosci Rep. 2005; 5(3):198-206.

21. Hegi ME, Diserens AC, Gorlia T, et al. MGMT gene silencing and benefit from temozolomide in glioblastoma. $N$ Engl J Med. 2005; 352(10):997-1003.

22. Carmeliet P. Angiogenesis in life, disease and medicine. Nature. 2005; 438(7070):932-936.

23. Kreisl TN, Kim L, Moore K, et al. Phase II trial of single-agent bevacizumab followed by bevacizumab plus irinotecan at tumor progression in recurrent glioblastoma. J Clin Oncol. 2009;27(5):740-745.
24. Vredenburgh JJ, Desjardins A, Herndon JE 2nd, et al. Bevacizumab plus irinotecan in recurrent glioblastoma multiforme. J Clin Oncol. 2007; 25(30):4722-4729.

25. Friedman HS, Prados MD, Wen PY, et al. Bevacizumab alone and in combination with irinotecan in recurrent glioblastoma. J Clin Oncol. 2009;27(28):4733-4740.

26. Lai A, Tran A, Nghiemphu PL, et al. Phase II study of bevacizumab plus temozolomide during and after radiation therapy for patients with newly diagnosed glioblastoma multiforme. J Clin Oncol. 2011;29(2): $142-148$.

27. Lucio-Eterovic AK, Piao Y, de Groot JF. Mediators of glioblastoma resistance and invasion during antivascular endothelial growth factor therapy. Clin Cancer Res. 2009;15(14):4589-4599.

28. Mellor HR, Callaghan R. Resistance to chemotherapy in cancer: a complex and integrated cellular response. Pharmacology. 2008;81(4): 275-300.

29. Bergers G, Hanahan D. Modes of resistance to anti-angiogenic therapy. Nat Rev Cancer. 2008;8(8):592-603.

30. Giantonio BJ. Targeted therapies: Goldie-Coldman and bevacizumab beyond disease progression. Nat Rev Clin Oncol. 2009;6(6):311-312.

31. Li X, Huang R, Xu Z. Risk of adverse vascular events in newly diagnosed glioblastoma multiforme patients treated with bevacizumab: a systematic review and meta-analysis. Sci Rep. 2015;5:14698.
OncoTargets and Therapy

\section{Publish your work in this journal}

OncoTargets and Therapy is an international, peer-reviewed, open access journal focusing on the pathological basis of all cancers, potential targets for therapy and treatment protocols employed to improve the management of cancer patients. The journal also focuses on the impact of management programs and new therapeutic agents and protocols on

\section{Dovepress}

patient perspectives such as quality of life, adherence and satisfaction. The manuscript management system is completely online and includes a very quick and fair peer-review system, which is all easy to use. Visit http://www.dovepress.com/testimonials.php to read real quotes from published authors. 